Voix et Images

voixetimages

\title{
Rétrospectives et prospectives
}

\section{André Brochu}

Volume 7, numéro 3, printemps 1982

Anne Hébert

URI : https://id.erudit.org/iderudit/200350ar

DOI : https://doi.org/10.7202/200350ar

Aller au sommaire du numéro

\section{Éditeur(s)}

Les Presses de l'Université du Québec

\section{ISSN}

0318-9201 (imprimé)

1705-933X (numérique)

Découvrir la revue

\section{Citer cet article}

Brochu, A. (1982). Rétrospectives et prospectives. Voix et Images, 7(3), 583-590.

https://doi.org/10.7202/200350ar d'utilisation que vous pouvez consulter en ligne.

https://apropos.erudit.org/fr/usagers/politique-dutilisation/ 


\title{
Rétrospectives et prospectives
}

\author{
par André Brochu, Université de Montréal
}

Grâce à un heureux concours de circonstances, j'inaugure cette chronique de poésie par la recension de plusieurs recueils de qualité. Je ne pourrai malheureusement m'étendre sur aucun; mais il est réjouissant de constater l'abondance et la diversité de la production poétique des quinze ou vingt derniers mois. L'essor du roman depuis le début des années 60 , du théâtre et de l'essai plus récemment, n'a pas nui à l'affirmation du genre poétique, que continuent d'illustrer des cuvres nouvelles et intéressantes'. La seule chose à regretter serait le manque croissant, proportionnellement. de lecteurs: de plus en plus de bons titres se disputent la faveur d'un nombre à peu près constant d'amateurs.

Je signale d'abord, de Rina Lasnier, Entendre l'ombre (poèmes) et Voir la nuit (proses) ${ }^{2}$, qui forment un diptyque. Le premier regroupe des poèmes brefs, le plus souvent composés de deux ou trois strophes, regroupés en quatre sections: Entendre l'ombre, Entendre le temps. Entendre l'amour et Entendre Dieu. Cette progression toute classique nous fait passer graduellement vers la lumière. d'emblée inscrite au cœur de l'ombre qui est dite "clairvoyante". Poésie dense, orante, qui approfondit avec méthode la méditation d'une vie vouée à la foi (qui est amour) et, simultanément, qui explore les ressources créatrices du langage, aux fins d'émerveillantes proférations:

Doucis-toi, doucis-toi...

défeuille-toi de turbulence

nudité d'excessive présence,

reprends ta robe ombrageante

doucie de fragile innocence...

Voir la nuit rassemble une vingtaine de textes de prose, selon une formule déjà utilisée dans Miroirs et l'Échelle des anges. ${ }^{3}$. L'inspiration religieuse y est omniprésente. Le lecteur sensible à la beauté de la poésie de Rina Lasnier y trouvera l'explication de plusieurs de ses thèmes et goûtera, avec un plaisir tout au moins intellectuel, son exégèse fine et bien personnelle des textes sacrés. 
De l"eil et de l'écoute. de Michel van Schendel4, sera pour plusieurs une révélation. Cette "rétrospective 1956-1976, qui reprend les deux recueils de 1958 (Poèmes de l'Amérique étrangère) et de 1964 (Variations sur la pierre). nous fait connaitre un grand nombre d'inédits. Elle révèle surtout la continuité d'une recherche, au long de vingt ans et plus de production poétique. Quelles quaient été les variations de thèmes et décriture, dictées par le souci d'accorder son engagement avec les inventions formelles les plus récentes. van Schendel garde une mème voix pressante, inquiète, chaleureuse, entrecoupée d'images rocailleuses, d'éclats de baguenaude, et puis d'injonctions graves comme la fin des temps. II magnifie la circonstance et fait, de cette pierre, son pain:

\section{Je ne crois qu'aux circonstances \\ Elles me sont chères \\ L'alpaga le jute et les cent rues aussi \\ L'emportent sur tout ce que tu aimes (...) \\ La circonstance détruit ce que tu dis qui n'y est pas}

Rien d'abstrait ni de compassé dans cette parole très construite, très tenue syntaxiquement mais aussi, vivante par l'élan qui la propulse, les images illuminantes.

En la nuit. la mer, deuxième "rétrospective" de Fernand Ouellette ${ }^{5}$, reprend le substantiel recueil publié en 1977, Ici, ailleurs, /a /umière ainsi que $A$ découvert (1979). Une section considérable s'y ajoute, qui donne son titre a l'ensemble. La thématique de "En la nuit, la mer» est plus unifiée que celle de «lci, ailleurs, la lumière», dont j'ai rendu compte en son temps ${ }^{6}$. Les poèmes regroupés sous le titre de "L'espace de la mer» nous présentent diverses images du bonheur, de l'ouverture de l'âme au corps, de soi à l'être aimé, et ce bonheur, cet accord avec l'autre font échec à la mort; inéluctable, celle-ci n'en sera pas moins dépassée:

Certes la mort va nous retourner entiers dans l'outre-visible, nous oublier ainsi qu'un vestige. un amas de blancheur mate:

mais le sourire poindra dans nos miembres.

Hélas, cette certitude n'est pas longtemps maintenue, et bientôt triomphe «Le temps de terrem, où sévit le «nocturne». Désormais, le bleu du jour est lui-mème mensonge: "en ce bleu tout est leurre. / ce bleu si bleu visage de la mort». L'espoir renaît difficilement de cette confrontation avec la nuit.

Tourné vers les sinistres réalités politiques de notre temps, le poète de "A découvert" retrouve sensiblement le même souffle, les mêmes rythmes étroits que pour parler de sujets plus personnels. Dans l'un et l'autre cas, 
la perspective est du reste la même, celle, pourrait-on dire, d'une métaphysique concrète où individualité et collectivité pèsent finalement d'un même poids. Jamais, chez Ouellette, on ne trouve d'expression pure et simple du moi: une quête de l'Être est sans cesse poursuivie, qui agrandit l'intime aux dimensions du monde. Inversement, les tragédies collectives ne sont abordées que sous l'angle restreint où l'individu peut les saisir - sans doute parce que l'individu, avec ses limites, son impuissance, est le seul sujet possible, en notre époque que l'épopée a désertée.

Desseins, de Michel Beaulieu?, constitue l'édition définitive de ses premiers poèmes (1961-1966). Des trois premiers recueils (Pour chanter dans les chaines. Ballades et satires et Le Pain quotidien), une trentaine de pièces seulement ont été retenues. Les recueils parus en 1966 et 1967 (Apatride, Merre et Érosions) sont repris intégralement. Des inédits s y ajoutent,

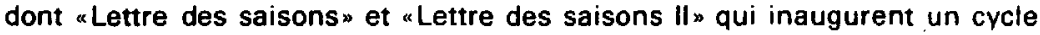
où vient s'inscrire le tout récent recueil, Visages ${ }^{8}$, qui en forme le cinquième et dernier volet.

"Cuvres de jeunesse", poèmes d'«apprentissage": c'est en ces termes que le poète nous présente sa rétrospective. Sans doute, la voix n'y a pas encore trouvé ses accents les plus personnels. II s'y trouve des réminiscences ( $" j$ jécris j'écris // ton nom sur le pin le sapin / le chêne rouge et le bouleau.... : on songe à Éluard et à Paul-Marie Lapointe) mais elles sont rares. II y a aussi de la prolixité. des facilités. Ce qui cependant traverse tous les textes, quelle qu'en soit l'inspıration, c'est une seule et mème volonté passionnée de faire le poème, d'être poète. Michel Beaulieu parle de tout et de rien, il n'a pas (encore) de thème bien à lui; mais il excelle à conférer un caractère de poésie à la moindre «idéen, par une disposition de mots toujours sûre. un métier. Poète de métier, voilà l'évidence que ces textes divers, jamais mauvais, nous communiquent quant à leur auteur.

Visages est aussi, à sa façon, une rétrospective puisqu'il rassemble cinq recueils dont un seul est inédit, les autres ayant paru en tirage limité ou ayant été lus à la radio. Cependant, toutes les pièces de ce substantiel recueil collectif ont été écrites en un laps de temps relativement court, de mars à décembre 1977. De là une grande unité d'inspiration. même si chaque groupe de poèmes a ses caractéristiques propres. "Zoo d'espèces", par exemple, pousse aussi loin que possible le recours à la parole quotidienne: " $($. . $)$ du vendeur $/$ qui a déménagé depuis le temps / mais pas aussi souvent que moi lain de là / de la Plaza Saint-Hubert à la Côte-de-Liesse / qui n'est pas plus gaie pour autant / comme on s'en doute / avec sa circulation de poids lourds / et ses nombreux avions / bien que le trafic en ait diminué / depuis l'ouverture de Mirabel». If s'agit là, heureusement, d'un cas limite. Dans "Personne», on trouve parfois, au contraire, un langage ciselé où les sonorités, par écho. multiplient les effets de sens: 


\author{
visage qu'épie l'épris \\ consumé dans ses plissements \\ parfois je te tue (l'as-tu. \\ ressenti \\ ce fouillement?) \\ vaguement pressenti
}

Les chaines signifiantes (épie - épris - plissements - ressenti pressenti; tue - tu - (ressen)ti) ensorcellent la parole, en font un chant du i en même temps que du visage aimé. Visage aimé en ses plis, précisément, car l'érotique de Beaulieu n'est jamais idéalisante, et la tendresse chez lui s'accompagne volontiers d'un réalisme cru, parfois jusqu'à l'outrance:

je remonte a l'amande amère et douce

qui durcit entre tes jambes

où t'en vas-tu quand ton vagin clapote

Les poèmes de « Neiges « et "Mai la nuit » disent, avec un lyrisme souvent touchant, les mouvements de l'âme quotidienne cherchant à naitre en la femme aimée et à se faire espace d'accueil pour que, en soi, elle naisse à son tour.

Ecrire, aimer. Ces deux mots, ensemble synonymes du mot vivre, forment le thème principal des trois textes, non à proprement parier des poèmes, que publie Jacques 8 rault sous le titre de Trois fois passera (précédé de Jour et nuit) ${ }^{9}$. «Fragments d'une lettre", "L'instant d'après "et "Une poétique en miettes» sont des textes de prose. Dans le premier s'insèrent des poèmes, sans titre et étroitement liés, par leur sujet, au contexte, de sorte qu'ils participent directement au développement de l'idée. Poésie et prose s'enchaînent, comme s'ils étaient de mème nature, que toute différence eût été abolie. C'est que la prose, malgré sa simplicité. y est d'une extrême rigueur. Aucun mot n'y est le fruit du hasard. Mais de la commune évidence, oui: "Je comprends enfin, grâce à cette songerie de choses banales, l'horreur chinoise et japonaise de l'originalité. La volonté de puissance a dévoyé l'écrire et l'aimer. d'arrière-pensée, avec laquelle Brault formule les lieux communs de l'existence et prend ainsi l'infini au piège du fini." "Je me sens bien dans nos lieux communs. C'est au fond du quotidien que git le merveilleux. II y en a qui se consacrent aux grandes choses - et je les admire; il y en a qui s'accordent avec les petites choses - et je les aime. " Une telle promotion du réel immédiat, non pas fermé sur soi mais ouvert sur la totalité du monde, rend seule plausible le passage, pour dire l'écrire-aimer, de la prose au poème, et l'inverse. En fin de compte, l'ouvrage de Brault, illustré de quatorze collages de Célyne Fortin et magnifiquement édité par le Noroît. s'adresse essentiellement au lecteur de poèmes et lui propose de riches méditations sur l'écriture, sur la femme, sur le vous 
Ion n'est pas très loin de la fin'amors\} qui, en amour, ase donne comme encore plus, et plus haut, et plus loin, et plus profond, et plus près aussi, que l'attendu, l'habituel nous."

Cette mise à distance du nous s'accompagne aussi du besoin de se faire étranger au pays, à soi-même: «Puisque toute poésie tend à devenir anonyme."

Il en va tout autrement chez Chamberland, qui a troqué le nous du pays pour une autre allégeance, spirituelle et cosmique, mais bien collégiale tout de même. Un texte de I'Enfant doré 10 évoque "ceux qui m'ont envoyé. missionné, qui de loin veillent sur moi / - mes guides, je distingue mal vos visages - ils se signalent habituellement comme un groupe, ils disent: «NOUS.. Le poète est l'envoyé des extraterrestres, un missionnaire, voire un messie (ce qui fait on ne peut plus québécois) et il a pour mandat de réaliser I'hommespèce en donnant naissance, par autoparthénogénèse, à l'hommenfant - si j'ai bien compris. L'opération est assez compliquée, et il faut une sensibilisation préalable à la gnose pour en goûter les détails. Tout cela relève du délire, un délire heureusement intelligent (à l'intérieur de ses limites) et cultivé (nourri de références à maints auteurs de la tradition occulte). un délire généreux aussi, jamais à court d'imagination ni, par delà les obsessions individuelles, de foi dans la possibilité de changer la vie et de rendre l'humanité vivable.

II me semble, du reste, que le délire tend, localement du moins, à reculer et faire place à une invention plus gratuite, une fantaisie qui avait disparu depuis longtemps de l'ceuvre de Chamberland. On trouve de nouveau de magnifiques et simples bonheurs d'expression, des vers qui sont comme de foudroyantes évidences de beauté:

quelle voracité vers le sable, l'eau nue

Je voudrais en citer d'autres... mais incapable d'en repérer, je constate à regret l'omniprésence de l'idée dans le texte.

Dans sa «poétique en miettes», Jacques Brault écrivait: "Les mots m'importent, certes, mais surtout leurs relations, le phrasé qui ouvre le vers a sa respiration profonde.» Gatien Lapointe, qui fait une rentrée après un silence de treize ans, semble renoncer au phrasé un peu solennel de r'Ode au Saint-Laurent pour explorer les joies primales, sinon primaires, du mot. Arbre-radar ${ }^{11}$, dont les poèmes furent écrits adu 9 février au 25 mars 1976» (année heureusement bissextile!), est une longue suite de salves verbales, de paysages dont on ne sait s'iis sont faits de mots ou de choses, peut-être parce qu'ils se situent dans un en-decà qui leur est commun. Une certaine 
posture adamique du poète subsiste, où les vocables ont remplacé les quadrupedes de l'Eden: «mots je vous touche et je vous nomme, les noms en premier, mottons de terre, bulbes d'étoiles (...) je vous jumelle, mots. je vous jette deux à deux, a trois, a neuf dans l'inaugural fracas de l'image». II y a du souffle, de l'homogénéité, de la pertinence dans ces textes qui, à certains égards, peuvent rappeler le Vierge incendié de Paul-Marie Lapointe mais sans leur variété ni, surtout, leur référence au réel-présent-concret. L'instrument privilégié de l'exploration verbale est la paronomase («rapprochement de mots dont le son est à peu près semblable, mais dont le sens est différent” - Littré) qui se présente souvent sous forme de mots attelés: roche/ruche. roué/troué, cor/corne, nerfs/nef, grotte/glotte, etc. On la rencontre aussi sous des formes plus libres: «jusqu'au chant, trébuchant hors de la mort". "outre du tain du lointain», "dans le roulis de fête dans le lit roux de la folie». Le rapprochement des mots est parfois futile: a dans le thorax de la nuit s'irradie le rose de l'ceil / / tout l'ceillet», parfois étonnamment suggestif: «enfer/ enfance, quoi gros cœur grogne muqueuse aurore?» Une nouvelle phrase est fondée, où l'homophonie tient lieu de tout: « qui d'éclairs glaire sucs sueurs d'arbre?" Les noms, on le voit, font la loi car il faudrait un sujet agissant pour justifier la présence de verbes.

Les thèmes d'autrefois surnagent, de loin en loin, mêlés aux mots fous: « natal pays nous (...) le monde descendant en nous posant en nous le nom neuf de chaque chose - habitable réel». Au centre du recueil, «Demeure de l'instant constate que les deux manières, l'ancienne et la nouvelle, font fort bon ménage.

Par la recherche d'Arbre-radar, Gatien Lapointe élargit son univers verbal, et peut-être bien son univers thématique. Ses prochains recueils devraient confirmer nos espoirs.

Le quotidien a deux couleurs possibles. Ou bien if est gris comme, par exemple, chez Michel Beaulieu: “cette grisaille au matin / bientôt le café l'exorcisera» (Visages). C'est le gris des choses, contre lequel on a de coutumiers recours. Ou bien il est, selon le titre du dernier recueil de Pierre Nepveu: Couleur chair'2. C'est la couleur du quotidien humain, lequel est bien plus redoutable que l'autre car on le sécrète soi-même, il est le monde tel que le fait notre pensée. C'est elle qui crée "la vague de réel" où elle se roule et s'angoisse à plaisir, où toute chose apparaît sous son aspect le plus défavorable:

les araignées dans la voix, le chaos dans les plis du sang. lumière obèse grignotant les joues, les assiettes de carton les os de poulet dans l'herbe entre juillet et l'âge de raison...

... forment le "tableau» (c'est le titre du poème) d'un coin d'existence où la désespérance disqualifie le jour et le corps même. Ailleurs, la pensée est 
comparée aux mouches "heurtant la vitre" et "incapables d'imaginer / une paroi qui ne soit / opaque» - bref, la pensée ne peut prendre son parti de l'impossibilité où elle est de sortir d'elle-même. Rien d'étonnant, dès lors, si « la maison de l'être est un taudis* (ô Heidegger!).

Le naturalisme poétique de Nepveu n'exclut pourtant pas le travaif de l'écriture, dont résultent de belles trouvailles:

téléphone proprement raccroché

sur un bouquet de raisons noires

Voué au réel, mais un réel qui n'a plus rien d'absolu et qui n'est peut-être que la projection, a l'extérieur, de son désabusement; maintenu pourtant bien vivant par une santé « fougueuse», qui détourne parfois le cours du poème dans le sens de l'espoir ( «La longue phrase déliée de la vie, à rebroussedétresse, trouve nos bouches chaque fois plus jeunes"), le poète dit peut-être la situation de l'homme privé de ses anciennes valeurs et impuissant à en créer de nouvelles. Dès lors, échappant à la juridiction du passé comme de l'avenir. il est voué au présent, "temps parfait" en son in-signifiance, temps neutre et nu (couleur chair): "Vivable damnation, degré zéro".

Combien dépouillée, mais aussi combien nécessaire apparaît la poésie de Gilbert Langevin dans ses deux derniers recueils, le fou solidaire et /ssue de secours ${ }^{13}$ ! Le premier surtout me semble exprimer tout l'essentiel d'une existence capable, malgré la souffrance, de vrai bonheur:

lois noires

et pourtant sous brise

fruits lumineux

Les poèmes réussissent en quelques mots à suggérer une étrange sagesse qui, loin de les exclure, assume la révolte et le devoir de rèver. La colère frappe peut-être un peu le vide quand elle s'en prend au «cartel à médailles», aux "hérodes" d'un Québec clérical, bien disparu maintenant. Mais quelle puissance d'évocation dans des vers comme les suivants:

il eut suffi de croire

petit halo falot

petit halo de feu battu

par un vlan de neige?

ou encore:

ingouvernable paix

parmi les rocs sauvages

La poésie de Langevin, comme les espèces d'hiéroglyphes qui illustrent Issue de secours, réalise le paradoxe d'un tracé signifiant très appuyé, fermé et comme pauvre; et pourtant étrangement ouvert à lui-même, à l'autre, à nous. 
Je garde pour une prochaine chronique plusieurs titres qui méritent plus qu'une simple mention.

A signaler, la parution d'une nouvelle revue de poésie, Urgences, qui publie des écrivains de l'Est du Québec. Deux numéros ont déjà paru. Aux éditions Passages de Rimouski, deux recueils. Westernité de Jean-Marc Cormier ${ }^{14}$ et La Tête dans le crin de Gilbert Depuis ${ }^{15}$, attestent la vitalité d'une littérature régionale qui ne se veut, d'aucune façon, régionaliste.

Enfin, au Noroit, un livre qui a peu à voir avec la poésie, et peut-ètre beaucoup tout de même: Soit dit en marchant... de Jacques Thisdel ${ }^{16}$. C'est une promenade hilarante, grâce aux talents graphiques de l'auteur, parmi les paysages de notre quotidienneté mystifiée.

1. Les aléas du service de presse expliquent l'absence de quelques noms considérables.

2. Rina Lasnier, Entendre I'ombre, vol. I, poèmes. Montréal, Hurtubıse HMH, 1981 : 84 p. Voir la nuit, vol. II, proses, Montréal, Hurtubise HMH, 1981; 165 p.

3. Miroirs, Montréal, l'Atelier, 1960: I'Echelle des anges, Montréal, Fides, 1975.

4. Michel van Schendel, De l'ceil et de l'écoute, poèmes 1956-1976, Montréal, I'Hexagone, 1980: $247 \mathrm{p}$. Ce livre a remporté le Prix du Gouverneur général.

5. Fernand Ouellette, En la nuit, la mer, poèmes 1972-1980, Montréal, l'Hexagone. $1981 ; 205 \mathrm{p}$.

6. "Fernand Ouellette, Ici, ailleurs, ta lumière", in Livres et auteurs québécors 1977. Québec. Presses de I'Université Laval, 1978; pp. 127-130.

7. Michel Beaulieu, Desseins, poèmes 1961-1966. Montréal, I'Hexagone, 1980: 246 p. Ce livre a remporté le Prix du Journal de Montréal.

8. Michel Beaulieu. Visages. Saint-Lambert. Le Noroit, 1981: $135 \mathrm{p}$.

9. Jacques Brault, Trors fois passera précédé de Jour et nuit, Saınt-Lambert, le Noroît, $1981: 87 \mathrm{p}$.

10. Paul Chamberland, L'Enfant doré, Montréal, l'Hexagone, $1981 ; 108 \mathrm{p}$.

11. Gatıen Lapointe, Arbre-radar, Montréal, l'Hexagone, 1980; 139 p.

12. Pierre Nepveu, Couleur chair, Montréal, l'Hexagone, 1980; 92 p.

13. Gilbert Langevin, Le Fou solidaire. Montréal, l'Hexagone, 1980; 66 p. Issue de secours, Montréal, I'Hexagone, 1981; 70 p.

14. Jean-Marc Cormier, Westernité (poèmes et chansons), Rimouski, passages, 1981 ; $90 \mathrm{p}$.

15. Gilbert Dupuis, La Tête dans le crin, Rimouski, Passages, 1981, $130 \mathrm{p}$.

16. Jacques Thisdel, Soit dit en marchant..., Saint-Lambert, Le Noroît, 1981 ; s.p. 